\title{
Correction to: Assessing the impacts of having a child with achondroplasia on parent well-being
}

Kathryn M. Pfeiffer ${ }^{1}(\mathbb{D}) \cdot$ Meryl Brod $^{1} \mathbb{D} \cdot$ Alden Smith $^{2} \mathbb{D} \cdot$ Jill Gianettoni $^{2} \cdot$ Dorthe Viuff $^{3} \cdot$ Sho Ota $^{2} \mathbb{D} \cdot$ R. Will Charlton ${ }^{2}$ iD

Published online: 30 October 2020

(c) The Author(s) 2020

\section{Correction to: Quality of Life Research https://doi.org/10.1007/s11136-020-02594-3}

In the original publication, the summary statistics for child BMI in Tables 2 and 8 were incorrect. The correct version of Tables 2 and 8 (with updated summary statistics) are provided in this correction.

The original article can be found online at https://doi.org/10.1007/ s11136-020-02594-3.

Meryl Brod

mbrod@ thebrodgroup.net

1 The Brod Group, 219 Julia Ave., Mill Valley, CA 94941, USA

2 Ascendis Pharma, Inc, Palo Alto, CA, USA

3 Ascendis Pharma, A/S, Hellerup, Denmark 
Table 2 Demographic/health characteristics for the children of parent participants

\begin{tabular}{|c|c|c|c|}
\hline & Spain $(n=11)$ & US $(n=25)$ & Total $(n=36)$ \\
\hline \multicolumn{4}{|l|}{ Child age, $n(\%)$} \\
\hline 2 to $<5$ years & $5(46)$ & $6(24)$ & $11(31)$ \\
\hline 5 to $<9$ years & $4(36)$ & $9(36)$ & $13(36)$ \\
\hline 9 to $<12$ years & $2(18)$ & $10(40)$ & $12(33)$ \\
\hline \multicolumn{4}{|l|}{ Child gender, $n(\%)$} \\
\hline Female & $7(64)$ & $12(48)$ & $19(53)$ \\
\hline Male & $4(36)$ & $13(52)$ & $17(47)$ \\
\hline \multicolumn{4}{|l|}{ Child's race/ethnicity, $n(\%)^{\mathrm{a}}$} \\
\hline Asian-American & - & 2(8) & - \\
\hline Black/African-American & - & $4(16)$ & - \\
\hline Latino/Hispanic & - & $2(8)$ & - \\
\hline White/Caucasian & - & $20(80)$ & - \\
\hline \multicolumn{4}{|l|}{$\begin{array}{l}\text { Age/time diagnosed with achondroplasia, } \\
n(\%)\end{array}$} \\
\hline In utero & $9(82)$ & $12(48)$ & $21(58)$ \\
\hline At birth & $1(9)$ & $4(16)$ & $5(14)$ \\
\hline$<2$ months of age & $1(9)$ & 2(8) & $3(8)$ \\
\hline $2-6$ months of age & 0 & $5(20)$ & $5(14)$ \\
\hline Unknown (adopted) & 0 & 2(8) & $2(6)$ \\
\hline $\begin{array}{l}\text { Child has parent(s) with achondroplasia, } \\
n(\%) \text { yes }\end{array}$ & 0 & $7(28)$ & $7(19)$ \\
\hline \multicolumn{4}{|l|}{ Health status (parent-reported), $n(\%)$} \\
\hline Excellent & $3(27)$ & $9(36)$ & $12(33)$ \\
\hline Very good & $3(27)$ & $11(44)$ & 14(39) \\
\hline Good & $3(27)$ & $4(16)$ & $7(19)$ \\
\hline Fair & $2(18)$ & 1(4) & $3(8)$ \\
\hline \multicolumn{4}{|l|}{ Height $(\mathrm{cm})$} \\
\hline Mean(SD) & $89.5(10.4)$ & $93.2(15.2)$ & $92.1(13.9)$ \\
\hline (Range) & $(75.0-104.0)$ & $(63.5-121.0)$ & $(63.5-121.0)$ \\
\hline \multicolumn{4}{|l|}{ Weight (kg) } \\
\hline Mean(SD) & 17.3(5.6) & $20.4(8.4)$ & 19.4(7.7) \\
\hline (Range) & $(10.0-28.0)$ & $(8.3-43.9)$ & $(8.3-43.9)$ \\
\hline \multicolumn{4}{|l|}{ BMI } \\
\hline Mean(SD) & $21.0(3.0)$ & $23.0(6.5)$ & $22.4(5.7)$ \\
\hline (Range) & $(16.7-26.9)$ & $(18.1-45.4)$ & $(16.7-45.4)$ \\
\hline $\begin{array}{l}\text { Planned limb lengthening surgery for the } \\
\text { future, } n(\%) \text { yes }\end{array}$ & $5(46)$ & 0 & $5(14)$ \\
\hline
\end{tabular}

Percentages may not add to 100 because of rounding

$S D$ standard deviation; $B M I$ body mass index

${ }^{a}$ US only; response categories are not mutually exclusive, so percentages do not add to 100 
Table 8 Cognitive debriefing parent participant and child demographic characteristics

\begin{tabular}{|c|c|c|c|}
\hline Parent participant demographics & & Child demographic/health characteristics & \\
\hline Parent age, mean(SD) & $39.1(6.4)$ & Child age, $n(\%)$ & \\
\hline (Range) & $(31-55)$ & 2 to $<5$ years & $4(25)$ \\
\hline Relationship to child, $n(\%)$ & & 5 to $<9$ years & $6(38)$ \\
\hline Mother & $16(100)$ & 9 to $<12$ years & $6(38)$ \\
\hline Parent has achondroplasia, $n(\%)$ yes & $2(12.5)$ & Child gender, $n(\%)$ & \\
\hline Marital status, $n(\%)$ & & Female & $12(75)$ \\
\hline Single & $1(6)$ & Male & $4(25)$ \\
\hline Married & $14(88)$ & Age/time diagnosed with achondroplasia, $n(\%)$ & \\
\hline No response & $1(6)$ & In utero & $5(31)$ \\
\hline Parent race/ethnicity, $n(\%)^{\mathrm{a}}$ & & At birth & $1(6)$ \\
\hline Asian-American & $1(6)$ & $<2$ months of age & $2(13)$ \\
\hline Latino/Hispanic & $1(6)$ & $2-6$ months of age & $5(31)$ \\
\hline White/Caucasian & $14(88)$ & $>6$ months of age & $1(6)$ \\
\hline Parent education, $n(\%)$ & & Unknown (adopted) & $2(13)$ \\
\hline High school or equivalent & $2(13)$ & Child's race/ethnicity, $n(\%)^{\mathrm{a}}$ & \\
\hline Vocational/technical school & $1(6)$ & Asian-American & $4(25)$ \\
\hline College degree & $9(56)$ & Latino/Hispanic & $1(6)$ \\
\hline Post-graduate school & $4(25)$ & White/Caucasian & 11(69) \\
\hline Parent work status, $n(\%)$ & & Child health status (parent-reported), $n(\%)$ & \\
\hline Full-time & $7(44)$ & Excellent & $8(50)$ \\
\hline Part-time & $4(25)$ & Very good & $7(44)$ \\
\hline Retired/student & 0 & Good & 0 \\
\hline Not working (other) & $5(31)$ & Fair & $1(6)$ \\
\hline Household income, $n(\%)$ & & Child height $(\mathrm{cm})$ & \\
\hline$<\$ 40,000$ & 0 & Mean(SD) & $90.8(12.0)$ \\
\hline$\$ 40,001$ to $\$ 60,000$ & $1(6)$ & (Range) & $(66.0-106.7)$ \\
\hline$\$ 60,001$ to $\$ 80,000$ & $1(6)$ & Child weight (kg) & \\
\hline$\$ 80,001$ to $\$ 100,000$ & $6(38)$ & Mean(SD) & $18.3(5.8)$ \\
\hline$>\$ 100,000$ & $6(38)$ & (Range) & $(10.9-31.7)$ \\
\hline \multirow[t]{3}{*}{ Decline to answer } & $2(13)$ & BMI & \\
\hline & & $\operatorname{Mean}(\mathrm{SD})$ & $21.8(3.8)$ \\
\hline & & (Range) & $(16.3-32.4)$ \\
\hline
\end{tabular}

$n=16$. Percentages may not add to 100 due to rounding

$S D$ standard deviation, $B M I$ body mass index

${ }^{a}$ Response categories are not mutually exclusive

Open Access This article is licensed under a Creative Commons Attribution 4.0 International License, which permits use, sharing, adaptation, distribution and reproduction in any medium or format, as long as you give appropriate credit to the original author(s) and the source, provide a link to the Creative Commons licence, and indicate if changes were made. The images or other third party material in this article are included in the article's Creative Commons licence, unless indicated otherwise in a credit line to the material. If material is not included in the article's Creative Commons licence and your intended use is not permitted by statutory regulation or exceeds the permitted use, you will need to obtain permission directly from the copyright holder. To view a copy of this licence, visit http://creativecommons.org/licenses/by/4.0/.

Publisher's Note Springer Nature remains neutral with regard to jurisdictional claims in published maps and institutional affiliations. 